Hong-Bo Tong* and Meng-Liang Wang

\title{
The crystal structure of bis $\left\{\left(\mu_{2}-3,3-\right.\right.$ dimethyl-1- phenylbut-1-en-2-yl)((dimethylamino) dimethylsilyl)amido- $\mathrm{K}^{3} N, N^{\prime}: N^{\prime}$ \}dilithium,
}

$\mathrm{C}_{32} \mathrm{H}_{54} \mathrm{Li}_{2} \mathrm{~N}_{4} \mathrm{Si}_{2}$

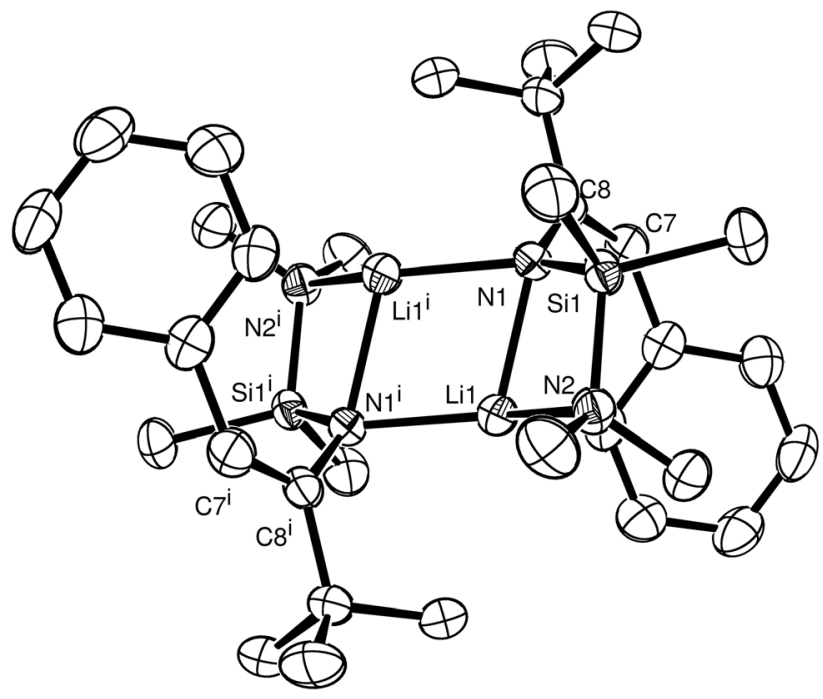

https://doi.org/10.1515/ncrs-2021-0090

Received March 14, 2021; accepted March 30, 2021;

published online April 14, 2021

\begin{abstract}
$\mathrm{C}_{32} \mathrm{H}_{54} \mathrm{Li}_{2} \mathrm{~N}_{4} \mathrm{Si}_{2}$, monoclinic, $P 2_{1} / n$ (no. 14), $a=11.611(4) \AA$, $b=11.157(6) \AA, c=13.307(5) \AA, \quad \beta=100.12(3)^{\circ}$, $V=1697.0(12) \AA^{3}, Z=2, R_{g t}(F)=0.0622, w R_{r e f}\left(F^{2}\right)=0.1561$, $\mathrm{T}=213 \mathrm{~K}$.
\end{abstract}

CCDC no.: 671708

The molecular structure is shown in the figure. Table 1 contains crystallographic data and Table 2 contains the list of the atoms including atomic coordinates and displacement parameters.

*Corresponding author: Hong-Bo Tong, Institute of Applied Chemistry, Shanxi University, Wucheng Rd. 92\#, Taiyuan, Shanxi Province, P. R. China, E-mail: tong@sxu.edu.cn. https://orcid.org/ 0000-0001-9222-9563

Meng-Liang Wang, Taiyuan Institute of Applied Chemistry, Shanxi University, Wucheng Rd. 92\#, Taiyuan, Shanxi Province, P. R. China
Table 1: Data collection and handling.

\begin{tabular}{ll}
\hline Crystal: & Colourless block \\
Size: & $0.30 \times 0.20 \times 0.20 \mathrm{~mm}$ \\
Wavelength: & Mo $K \alpha$ radiation $(0.71073 \AA)$ \\
$\mu:$ & $0.13 \mathrm{~mm}^{-1}$ \\
Diffractometer, scan mode: & Bruker SMART APEX II, $\varphi$ and $\omega$ \\
$\theta_{\text {max }}$, completeness: & $25.0^{\circ},>99 \%$ \\
$N(h k l)_{\text {measured }}, N(h k l)_{\text {unique }}, R_{\text {int }}:$ & $6768,2982,0.024$ \\
Criterion for $I_{\text {obs }}, N(h k l)_{\text {gt }}:$ & $I_{\text {obs }}>2 \sigma\left(I_{\text {obs }}\right), 2878$ \\
$N(\text { param })_{\text {refined }}:$ & 199 \\
Programs: & Siemens [1], ORTEP-3 [2], SHELX \\
& {$[3-5]$} \\
\hline
\end{tabular}

\section{Source of material}

All manipulations were carried out using standard Schlenk and glovebox techniques under an argon or nitrogen atmosphere. The title compound was synthesized according to literature methods [6-8]. To a solution of ((dimethylamino)dimethylsilyl)toluollithium $(6 \mathrm{mmol})$ in diethyl ether(20 ml), tert-butyl nitrile (6 mmol) was added at $c a$. $273 \mathrm{~K}$ and the solution was stirred for $15 \mathrm{~min}$ and then kept stirring for $5 \mathrm{~h}$ at room temperature. The solvent was slowly concentrated under vacuum, crystals of the title compound were obtained within two days.

\section{Experimental details}

Coordinates of hydrogen atoms were refined without any constraints or restraints. Their $U_{\text {iso }}$ values were set to $1.2 U_{e q}$ of the parent atoms.

\section{Comment}

Amidinate compounds are well known and have played important role in searching for novel spectator ligands $[6-8,13]$. Lappert and co-workers have prepared a variety of complexes including some main group elements and transition metals [9-12]. Quite a long time ago we have got similar complexes [14, 15]. As part of an subsequent 
Table 2: Fractional atomic coordinates and isotropic or equivalent isotropic displacement parameters $\left(\AA^{2}\right)$.

\begin{tabular}{|c|c|c|c|c|}
\hline Atom & $x$ & $y$ & $z$ & $U_{\text {iso }} * / U_{\text {eq }}$ \\
\hline Li1 & $0.5090(4)$ & $0.0789(4)$ & $0.0653(3)$ & (10) \\
\hline N1 & $0.36147(18)$ & 0.02269 (18) & $0.00870(16)$ & $0.0262(5)$ \\
\hline N2 & 0.45798 (19) & $0.0373(2)$ & $0.20620(16)$ & $0.0312(5)$ \\
\hline Si1 & $0.34215(6)$ & $-0.04527(6)$ & $0.13059(6)$ & $0.0272(2)$ \\
\hline $\mathrm{C} 1$ & 0.2707 (3) & $0.2271(2)$ & $-0.0360(2)$ & $0.0340(6)$ \\
\hline$C 2$ & $0.1946(3)$ & $0.3073(3)$ & $-0.0014(2)$ & $0.0441(7)$ \\
\hline $\mathrm{H} 2$ & 0.114229 & 0.289556 & -0.011757 & $0.053^{*}$ \\
\hline C3 & $0.2343(3)$ & $0.4125(3)$ & $0.0479(3)$ & $0.0512(9)$ \\
\hline H3 & 0.180700 & 0.465128 & 0.070118 & $0.061^{*}$ \\
\hline $\mathrm{C} 4$ & $0.3511(3)$ & 0.4407 (3) & $0.0647(3)$ & 0.0498 (8) \\
\hline H4 & 0.377999 & 0.511409 & 0.099518 & $0.060^{*}$ \\
\hline $\mathrm{C} 5$ & $0.4287(3)$ & $0.3641(3)$ & $0.0299(2)$ & $0.0430(7)$ \\
\hline H5 & 0.508812 & 0.383099 & 0.040122 & $0.052^{\star}$ \\
\hline C6 & $0.3886(3)$ & $0.2592(2)$ & $-0.0200(2)$ & $0.0360(7)$ \\
\hline H6 & 0.442412 & 0.208271 & -0.043872 & $0.043^{*}$ \\
\hline $\mathrm{C} 7$ & $0.2258(2)$ & $0.1167(3)$ & $-0.0896(2)$ & $0.0355(6)$ \\
\hline $\mathrm{H} 7$ & 0.162078 & 0.125252 & -0.143484 & $0.043^{*}$ \\
\hline $\mathrm{C} 8$ & $0.2666(2)$ & $0.0048(2)$ & $-0.0693(2)$ & $0.0296(6)$ \\
\hline C9 & 0.2019 (2) & $-0.1010(3)$ & $-0.1301(2)$ & $0.0376(7)$ \\
\hline C10 & 0.1335 (3) & $-0.0627(4)$ & -0.2349 & $0.0578(10)$ \\
\hline $\mathrm{H} 10 \mathrm{~A}$ & 0.185061 & -0.019013 & -0.271899 & $0.087^{*}$ \\
\hline H10B & 0.068690 & -0.011671 & -0.225411 & $0.087^{\star}$ \\
\hline $\mathrm{H} 10 \mathrm{C}$ & 0.103798 & 289 & -0.273532 & $0.087^{\star}$ \\
\hline C11 & $0.1145(3)$ & $-0.1557(3)$ & $-0.0684(3)$ & $0.0508(9)$ \\
\hline $\mathrm{H} 11 \mathrm{~A}$ & 0.072321 & -0.220685 & -0.106995 & $0.076^{*}$ \\
\hline $\mathrm{H} 11 \mathrm{~B}$ & 0.059627 & -0.094604 & -0.055308 & $0.076^{*}$ \\
\hline $\mathrm{H} 11 \mathrm{C}$ & 0.156609 & -0.186287 & -0.004093 & $0.076^{*}$ \\
\hline $\mathrm{C} 12$ & $0.2888(3)$ & $-0.1986(3)$ & $-0.1472(3)$ & 0.0509 (9) \\
\hline C13 & $0.1986(2)$ & 0.0090 (3) & $0.1588(2)$ & $0.0395(7)$ \\
\hline H13A & 0.177577 & 82 & 0.122537 & $0.059^{*}$ \\
\hline $\mathrm{H} 13 \mathrm{~B}$ & 0.205212 & 0.022378 & 0.231567 & $0.059^{\star}$ \\
\hline $\mathrm{H} 13 \mathrm{C}$ & 0.138785 & -0.050680 & 0.136753 & $0.059^{*}$ \\
\hline C14 & 0.3595 (3) & $-0.2044(3)$ & $0.1753(3)$ & $0.0436(7)$ \\
\hline $\mathrm{H} 14 \mathrm{~A}$ & 0.297399 & -0.252848 & 0.137145 & $0.065^{*}$ \\
\hline H14B & 0.355473 & -0.208114 & 0.247413 & $0.065^{*}$ \\
\hline $\mathrm{H} 14 \mathrm{C}$ & 0.434654 & -0.234776 & 0.164459 & $0.065^{*}$ \\
\hline C15 & $0.4246(3)$ & 0.1518 & $0.2479(2)$ & $0.0418(7)$ \\
\hline $\mathrm{H} 15 \mathrm{~A}$ & 0.388402 & 0.136531 & 0.307042 & $0.063^{*}$ \\
\hline H15B & 0.369704 & 0.193570 & 0.196358 & $0.063^{*}$ \\
\hline $\mathrm{H} 15 \mathrm{C}$ & 0.493833 & 0.200914 & 0.267952 & $0.063^{*}$ \\
\hline C16 & 0.5414 (3) & $-0.0256(3)$ & $0.2841(2)$ & 0.0482 (8) \\
\hline $\mathrm{H} 16 \mathrm{~A}$ & 0.604663 & 0.028226 & 0.311720 & $0.072^{\star}$ \\
\hline $\mathrm{H} 16 \mathrm{~B}$ & 0.572831 & -0.094484 & 0.253573 & $0.072^{*}$ \\
\hline $\mathrm{H} 16 \mathrm{C}$ & 0.501790 & -0.052198 & 0.338534 & $0.072^{\star}$ \\
\hline $\mathrm{H} 12 \mathrm{~A}$ & 0.347 (3) & $-0.168(3)$ & $-0.188(2)$ & $0.046(9)^{\star}$ \\
\hline H12B & $0.246(3)$ & $-0.267(3)$ & $-0.182(3)$ & $0.062(10)^{\star}$ \\
\hline $\mathrm{H} 12 \mathrm{C}$ & $0.332(3)$ & $-0.230(3)$ & $-0.079(3)$ & $0.057(10)^{*}$ \\
\hline
\end{tabular}

investigation of the chemical and physical properties of amidinate complexes, we have prepared the title complex, and present its structure here (the Figure).
The title compound is a centrosymmetric dimer which contains two NSiN fragments bonded to two central Li atoms. Thus it is a good spectator ligand to study its conjugation and chemical properties. The two amidinate ligands bound to two $\mathrm{Li}$ atoms, with $\mathrm{Li}-\mathrm{N}$ bond lengths 2.081(5), 2.114(5) and 2.036(5) $\AA$. The central Li atoms are part of three planes: two crystallographically identical fourmembered planes (N1/Si1/N2/Li1 with RMS deviation $=0.0111 \AA)$ and one central four-membered ring $(\mathrm{N} / \mathrm{Li}$ with symmetry). The former plane encloses an angle of $45.7(3)^{\circ}$ with the latter plane. And these three planes form a cascade-step configuration. Each lithium atom is surrounded by three nitrogen atoms. Some slightly different Li complexes have been found with similar configuration which have similar manner bonded to central Li atoms $[8,12,14,16]$.

Author contributions: All the authors have accepted responsibility for the entire content of this submitted manuscript and approved submission.

Research funding: We gratefully acknowledge support by Key research and development projects in Shanxi Province No. 201803D121040 for financial support.

Conflict of interest statement: The authors declare no conflicts of interest regarding this article.

\section{References}

1. Siemens. SMART and SAINT; Siemens Analytical X-ray Instruments Inc.: Madison Wisconsin, USA, 1996.

2. Farrugia L. J. ORTEP-3 for Windows-a version of ORTEP-III with a graphical user interface (GUI). J. Appl. Crystallogr. 1997, 30, 565.

3. Sheldrick G. M. SAINT (Version 6.02), SADABS (Version 2.03); Bruker AXS Inc.: Madison, 2002.

4. Sheldrick G. M. SHELXTL - integrated space-group and crystalstructure determination. Acta Crystallogr. 2015, A71, 3-8.

5. Sheldrick G. M. Crystal structure refinement with SHELXL. Acta Crystallogr. 2015, C71, 3-8.

6. Hitchcock P. B., Lappert M. F., Layh M., Liu D.-S., Sablong R., Shun J. Reactions of $\mathrm{LiCHR}_{2}$ and related lithium alkyls with $\alpha-\mathrm{H}$ free nitriles and the crystal structures of eleven representative lithium 1,3-diazaallyls, 1-azaallyls and $\beta$-diketiminates. J. Chem. Soc. Dalton Trans. 2000, 2301-2312; https://doi.org/10.1039/ b002376k.

7. Hitchcock P. B., Lappert M. F., Wei X.-H. Synthesis and structures of crystalline lithium 1-azaallyls and a 1,3-diazaallyl derived from $\mathrm{Li}$ $\left\{\mathrm{CH}\left(\mathrm{SiMe}_{3}\right)\left(\mathrm{SiMe}_{3^{\prime} n}(\mathrm{OMe})_{n}\right)\right\}(n=1$ or 2$)$ and $\mathrm{Li}\left\{\mathrm{CH}\left(\mathrm{SiMe}_{2} \mathrm{OMe}\right)_{2}\right\}$ and $\mathrm{RCN}\left(\mathrm{R}={ }^{t} \mathrm{Bu}, \mathrm{Ph}, 2,5-\mathrm{Me}_{2} \mathrm{C}_{6} \mathrm{H}_{3}\right.$, or Ad). J. Organomet. Chem. 2003, 683, 83-91.

8. Avent A. G., Hitchcock P. B., Lappert M. F., Sablong R., Severn J. R. Synthesis, structures, characterization, dynamic behavior, and reactions of novel late transition metal(II) 1-azaallyls. Organometallics 2004, 23, 2591-2600. 
9. Blystone S. L. Synthetic applications of enantioselective organotransition-metal-mediated reactions. Chem. Rev. 1989, 89, 1663-1679.

10. Barker J., Kilner M. The coordination chemistry of the amidine ligand. Coord. Chem. Rev. 1994, 133, 219-300.

11. Edelmann F. T. N-silylated benzamidines: versatile building blocks in main group and coordination chemistry. Coord. Chem. Rev. 1994, 137, 403-481.

12. Kissounko D. A., Zabalov M. V., Brusova G. P., Lemenovskii D. A. Principal trends in the chemistry of amidinate complexes of maingroup and transition elements. Russ. Chem. Rev. 2006, 75, 351-374.

13. Caro C. F., Lappert M. F., Merle P. G. Review of metal 1-azaallyl complexes. Coord. Chem. Rev. 2001, 219-221, 605-663.
14. Yuan S.-F., Wei X.-H., Tong H.-B., Zhang L.-P., Liu D.-S., Sun W.-H. $\mu_{2}, \eta^{1}-N-[(N, N$-Dimethylamino)dimethylsilyl]-2,6-diisopropylanilido metal ( $\mathrm{Li}, \mathrm{Zr}, \mathrm{Hf}$ ) compounds and the catalytic behaviors of the IVB compounds in ethylene (co)polymerization. Organometallics 2010, 29, 2085-2092.

15. Yuan H.-Y., Tong H.-B., Wei X.-H. Bis( $\eta^{3}$-2-tert-butyl-3-phenyl1-trimethylsilyl-1-azaallyl)cobalt(II). Acta Crystallogr. 2007, E63, m1325-1327.

16. Bai S.-D., Guo J.-P., Liu D.-S. A convenient route to silyl linked bis(amidinate) ligands and synthesis of group(i) metal derivatives. Dalton Trans. 2006, 2244-2250; https://doi.org/10. 1039/B516283A. 